Übergewicht beginnt bei einem BodyMass-Index (BMI) von 25, Fettleibigkeit ab 30. Das entspricht einem Gewicht von 98 Kilo bei einer Körpergröße von 180 Zentimetern. Gemäß der WHO-Prognose wären in 15 Jahren fast jeder vierte Mann und gut jede fünfte Frau fettleibig. „Wir brauchen klare und evidenzbasierte Konzepte, mit denen wir dieser fatalen Entwicklung erfolgreich entgegensteuern können“, erklärt DGVS-Sprecher Professor Christian Trautwein, Direktor der Klinik für Gastroenterologie, Stoffwechselerkrankungen und Internistische Intensivmedizin (Medizinische Klinik III) an der Uniklinik RWTH Aachen.

Denn die Folgen für den Stoffwechsel sind erheblich, für die Patienten mitunter sogar tödlich. Bei bis zu 90\% der stark übergewichtigen Menschen verfettet die Leber. Häufig entzündet sich das Organ und es entwickelt sich eine Fettlebererkrankung. Wird diese nicht frühzeitig behandelt, kann aus der Hepatitis eine Leberzirrhose und Leberkrebs hervorgehen. Eine Zunahme um 5 Einheiten des BMI erhöht das
Risiko für ein Leberzellkarzinom um durchschnittlich 39\%. „Gerade ist eine neue DGVS-Leitlinie erschienen, an der sich Ärzte bei der Behandlung der NichtAlkoholischen Fettlebererkrankung orientieren können“, sagt Trautwein. Neben der Entwicklung wissenschaftlich basierter Behandlungsempfehlungen sei es jedoch notwendig, noch mehr in die Präventionsforschung zu investieren, ergänzt der Experte. So zeichne sich beispielsweise in den letzten Jahren ab, dass die Zusammensetzung der Darmflora das Gewicht beeinflusst. „Studien deuten darauf hin, dass Störungen des Mikrobioms die Neigung zu Übergewicht fördern“, erklärt Trautwein. Kinder etwa, die während der ersten 5 Lebensmonate Antibiotika bekommen, sind eher von Übergewichtig betroffen. Umgekehrt scheint eine Veränderung der Darmflora auch Übergewicht und Krankheiten wie Typ-2-Diabetes zu bessern. „Solche Zusammenhänge müssen detaillierter untersucht und die Funktionen einzelner Stämme von Darmbakterien geklärt werden“, sagt Trautwein.

\title{
DDG kritisiert Forsa-Umfrage der DAK als irreführend
}

Nichtübertragbare Krankheiten wie Typ2-Diabetes, Übergewicht und Herz-Kreislauferkrankungen verursachen weltweit rund $80 \%$ aller Todesfälle. Auch die volkswirtschaftlichen Schäden sind enorm: Allein die direkten Kosten des Diabetes in Deutschland werden auf über 22 Milliarden Euro geschätzt. Viele Politiker und Vertreter des Gesundheitswesens fordern daher eine Steuer auf kalorienreiche Nahrungsmittel, da Übergewicht chronische Erkrankungen stark begünstigt. Nun ermittelte eine Forsa-Umfrage im Auftrag der DAK, dass nur 30\% der Bevölkerung eine solche Besteuerung für geeignet halten, Menschen vor Erkrankungen zu schützen. „Wir halten diese Umfrage für irreführend und nicht aussagekräftig“, konstatiert Privatdozent Dr. med. Erhard Siegel, Präsident der DDG.

So wurde den Befragten laut Untersuchungsergebnissen der Umfrage der wich- tige zweite Teil einer Zucker/Fettsteuer verschwiegen, nämlich eine entsprechende Steuerentlastung zugunsten gesunder und kalorienärmerer Lebensmittel.

„Offensichtlich ist die andere - durchaus attraktive - Seite der Medaille bei der Forsa-Umfrage unter den Tisch gefallen. Daher müssen wir hier von einer Irreführung der Befragten ausgehen, einer geschickten Manipulation“, kritisiert Dr. Dietrich Garlichs, Geschäftsführer der DDG. Mit Sicherheit würden einer Zucker/ Fettsteuer mehr Menschen zustimmen, wenn ihnen im Gegenzug eine finanzielle Entlastung angeboten würde.

„Ein Preissignal könnte der Bevölkerung helfen, gesündere Kaufentscheidungen zu treffen und der Lebensmittelindustrie Anreize bieten, gesündere Produkte zu entwickeln. Denn bisherige Appelle an die Vernunft des Einzelnen seien angesichts des Tsunamis der chronischen
Krankheiten nachweislich gescheitert“, erklärte Garlichs.

Bemerkenswert an der Umfrage ist, dass die große Mehrheit der Befragten (85\%) eine Ampelkennzeichnung auf Lebensmittelverpackungen als geeignete Maßnahme gegen Übergewicht und Adipositas ansehen, ebenso wie ein Werbeverbot für Kinderlebensmittel (65\%). Überraschend ist auch, dass 49\% der Meinung sind, dass es zu den Aufgaben der Politik gehöre, sich um die Ernährungsgewohnheiten der Bürger zu kümmern. Die Zustimmung ist besonders hoch unter den Jüngeren und besser Gebildeten. Nur 43\% finden, dass die Politik sich aus dem Bereich heraushalten sollte. Forsa-Umfrage zur Zuckersteuer: http://www.dak.de/ dak/download/Forsa-Umfrage_zur_Zuckersteuer-1616700.pdf?

Pressemitteilung DDG, 12.5.2015 